\title{
PHOTOGRAMMETRIC PERFORMANCE OF AN ULTRA LIGHT WEIGHT SWINGLET "UAV"
}

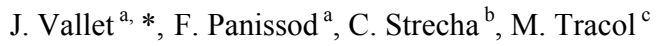 \\ ${ }^{a}$ Helimap System SA, Le grand-chemin 73, 1066 Epalinges, Switzerland - (julien.vallet,flory.panissod)@helimap.ch \\ ${ }^{\mathrm{b}}$ Pix4D, Chemin des Clos 117b, 1024 Ecublens, Switzerland - info@pix4d.com \\ ${ }^{\mathrm{c}}$ ENSA-Strasbourg, 24 Bld de la Victoire 67084 Strasbourg Cedex, France - matthieu.tracol@insa-strasbourg.fr
}

KEY WORDS: UAVs, Ultra light, Photogrammetry, aerial triangulation, camera, calibration, DTM, orthophoto.

\begin{abstract}
:
Low cost mapping using UAV technology is becoming a trendy topic. Many systems exist where a simple camera can be deployed to take images, generally georeferenced with a GPS chip and MEMS attitude sensors.

The step from using those images as information picture to photogrammetric products with geo-reference, such as digital terrain model (DTM) or orthophotos is not so big. New development in the field of image correlation allow matching rapidly and accurately images together, build a relative orientation of an image block, extract a DTM and produce orthoimage through a web server.

The following paper focuses on the photogrammetric performance of an ultra light UAV equipped with a compact 12Mpix camera combined with online data processes provided by Pix4D.

First, the step of image orientation is studied with the camera calibration step, thus the DTM extraction will be compared with conventional results from conventional photogrammetric software, new generation technique of pixel correlation and with reference data issued from high density laser scanning. The quality of orthoimage is presented in terms of quality and geometric accuracy.
\end{abstract}

\section{INTRODUCTION}

Unmanned Airborne Vehicles (UAV) has a long history in the military applications. The upcoming of those technologies to civil domains for non "high skills" pilot is very recent. The mapping field is not an exception. Drones were highly used for such purpose in military operations during last decades.

Since mid 2000's, research focused on the use of such vehicle to acquire low cost mapping datasets, mainly based on imagery and georeferencing sensors (Wendel et al., 2006). As the payload is the crucial point, the miniaturization of the sensors during the last years permitted to either use lighter vehicle or to bring more or higher grade sensors to a given platform. In parallel, the release of miniaturized georeferencing sensors such as GPS chip or inertial micro-electromechanical systems (MEMS) gave the possibility to pilot automatically the vehicle and to geo-reference acquired data (Eisenbeiss et al. 2009).

Due to the payload limitation, acquired datasets consists mostly in digital images, generally associated with a GPS position and sometimes attitude information.

Starting from this, it is possible using photogrammetric process to first get the orientation of the images and thus to extract digital terrain/surface model and Orthophoto.

The quality of the resulting 3D data will depend on geometric and radiometric image quality. Light digital cameras have not been design for photogrammetric purpose but the new developments in multi-image correlation are in a way to change drastically the world of photogrammetry (Haala, 2009).

The goal of this paper is to examine the potential of such low cost mapping in terms of photogrammetric accuracy for resulting products: Digital Terrain/Surface Model (DTM/DSM) and Orthophoto. The experiences were led using an ultra light weight UAV with a compact digital camera. Different photogrammetric processes are approached and compared to a reference dataset issued from high accuracy LiDAR/photogrammetric flight and ground measurements. A special attention is drawn to the analysis of the internal geometry of the camera and its consequences to DTM/orthophoto.

\section{EQUIPMENT AND METHODOLOGY}

\subsection{Acquisition system description}

\subsubsection{The vehicle}

The acquisition system is based on a very light weight UAV, weighting less than $500 \mathrm{~g}$. The system is called "Swinglet" from Sensefly and is composed of a light wingspan combined with a u-BLOX GPS chip, an attitude sensor, a radio transmitter and an autopilot circuit board (fig. 1). The maximum payload is 125g. An autopilot allows driving the UAV automatically on the flight lines and triggering the pictures. Power supply is assured with a small Lithium-Ion battery and flight autonomy is about $30 \mathrm{~min}$.

The Swinglet can operate only in low wind (less than $20 \mathrm{~km} / \mathrm{h}$ ).

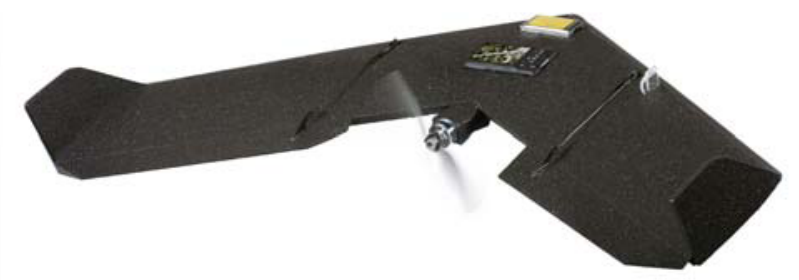

Figure 1: The Swinglet from Sensefly. The wingspan is only $80 \mathrm{~cm}$ and the total weight including sensing device is $500 \mathrm{~g}$.

\subsubsection{The image sensor}


The camera used is a Canon Ixus 120IS with 12 Megapixel (4000x3000 pixels). The CCD size is $6.16 \times 4.62 \mathrm{~mm}$ with a pixel size of $1.54 \mu \mathrm{m}$. The focal length varies from $5 \mathrm{~mm}$ to 20 $\mathrm{mm}$.

The camera setup for data acquisition is managed automatically with autofocus and automatic speed-aperture settings. To protect the camera during take off and landing, the camera is shutdown during those steps.

Images are triggered automatically at constant interval for the tested version. The latest firmware provides a more flexible trigger function of flight height above ground, ground velocity and expected overlap.

\subsubsection{Georeferencing}

One GPS chip provides a navigation position based on C/A code each second but no raw data of code is recorded (for a possible post-processing). Each image is tagged with GPS position stored in the EXIF data.

A small attitude sensor provides the three orientation angles roll, pitch and heading. Both datasets are used to drive the Swinglet on the planned lines and the resulting trajectory is recorded at $1 \mathrm{~Hz}$ (Zuffrey et al. 2010).

\subsection{Data processing}

Two processing workflows have been run in parallel. One using classical photogrammetric tools and the second one using a modern approach from computer vision science: dense image matching.

\subsubsection{Classical Photogrammetric process}

A classical photogrammetric workflow has been used here to treat the images consisting of performing an aerial triangulation and a bundle adjustment to compute photo orientation and camera calibration. Thus, a DSM extraction and an orthomosaic have been computed. SocetSet 5.5 from BAE Systems and Bingo-F has been used for photogrammetric process and Bundle adjustment.

The focal length, principal point and radial/tangential distortions were set as unknown in the Bundle adjustment to determine the internal orientation parameters according to the Bingo-F model (Bingo Manual).

A dozen of Ground Control Points (GCP's) were used too.

The DSM extraction has been computed with the NGATE module of SocetSet which is based on multi-image correlation (Ngate documentation).

\subsubsection{Dense Image matching}

The second workflow is a more automated process based on the dense image matching technology (Tola and al. 2010). A complete automated integration of tie points measurements, camera calibration, DSM extraction and true orthomosaic production has been implemented through a web based interface by Pix4D.

Georeference of the EXIF tags is used to provide absolute reference to the image bloc with an accuracy of GPS navigation chip (few meters). After this step, it is possible to include GCP's to refine absolute orientation of the bloc.

The camera calibration is modelled similarly to Matlab camera toolbox parameters (3 for radial and 2 for tangential). More details on this workflow are presented by Küng (Küng, 2011)

\subsection{Analysis Methodology}

For each type of workflow, resulting data are composed of:

- camera calibration parameters

- Bundle adjustment results: estimated tie points coordinates

- DTM/DSM

- $\quad$ Orthomosaic

Those data were compared to reference data issued from LiDAR-photogrammetric flight with Helimap System. High resolution DTM/DSM (10 pt/m²), 22 megapixel images and 12 additional GCP's measured in the studied area compose the reference dataset.

\subsubsection{Bundle adjustment and camera calibration}

As both processes use different camera models, it is hard to compare methods together. The analysis focuses more on the results achieved by each process. A special attention is drawn to camera calibration results especially for non radial lens distortion.

\subsubsection{DTM/DSM comparison}

DTM/DSM's have been compared to reference LiDAR DTMDSM and to GCP's.

The DTM/DSM comparison has been made by differencing regular grids. The grids with a post spacing of $25 \mathrm{~cm}$, are derived from triangular network (TIN) of the original points.

The absolute vertical accuracy was achieved with GCP projection on the TIN model.

\subsubsection{Orthophoto comparison}

The orthomosaic accuracy is performed by comparing firstly the GCP position to get absolute planimetric accuracy. Secondly, the orthomosaic from both workflows are compared to the reference orthophoto by digitizing ground elements such as painted marks on ground, manholes. No comparison where made on surface structures (building).

\section{EXPERIMENTS AND ANALYSIS}

\subsection{Flights}

\subsubsection{Swinglet flight}

Experimental flights were conducted the $5^{\text {th }}$ of March 2011 with the Swinglet over the Helimap calibration field. A classical calibration flight line "cross" pattern was flown at 100m and $150 \mathrm{~m}$ above ground level (fig. 2). This leads to a ground sample dimension of $\sim 4 \mathrm{~cm}$. The overlap is extremely variable since the triggering was only possible at constant interval and depending on the wind direction. Overlap varies from $50 \%$ to $80 \%$ with sometime a lot of drift in heading (fig. 3). 


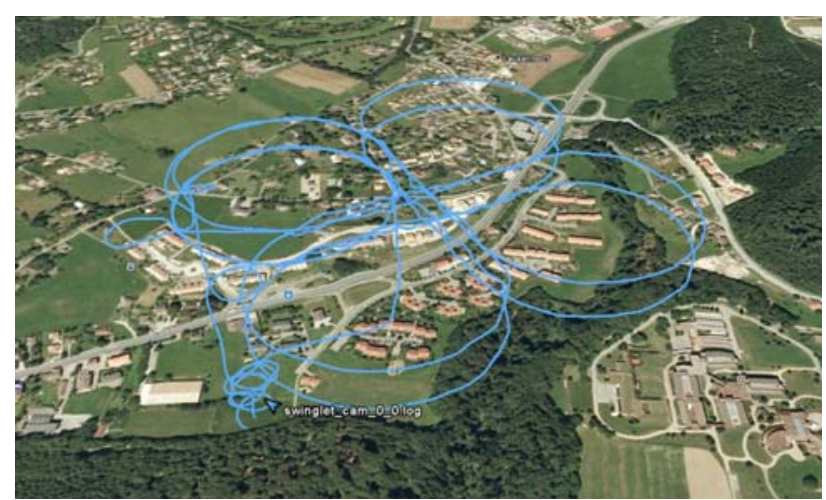

Figure 2: The trajectory of the Swinglet flight. The waves on the flight lines are due to alternative switched of the propeller, on to move, off during the camera trigger. This is done to increase stability during the image shot.

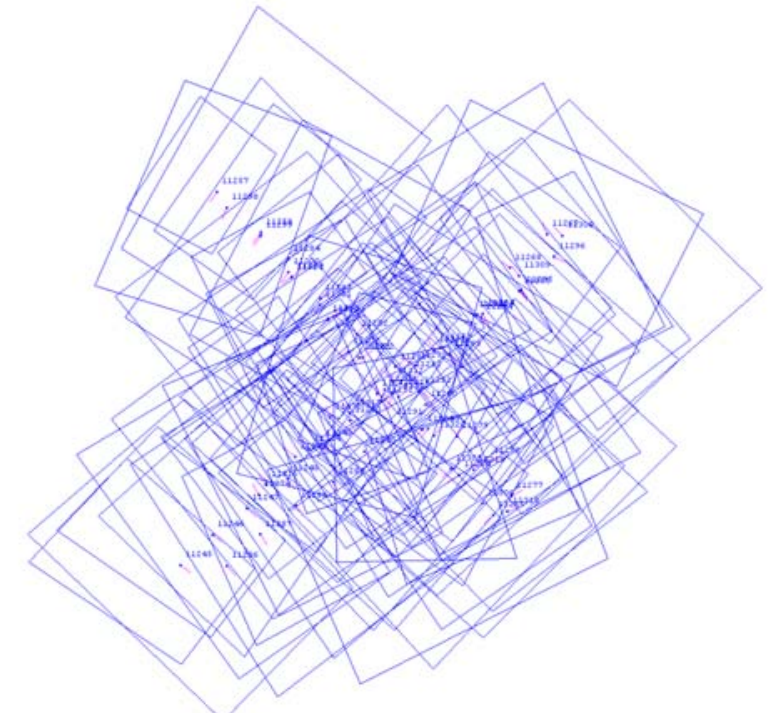

Figure 3: Footprint of the images of the experimental flight. In the centre of the cross, points are visible on more than 30 images. Heading of the images varies a lot in flightlines due to the wind.

\subsubsection{Reference data}

A similar flight was conducted on $14^{\text {th }}$ of June 2011 with Helimap System (Vallet, 2007) with a flying height of $200 \mathrm{~m}$ and $250 \mathrm{~m}$ above ground level. Ground pixel size is $\sim 5 \mathrm{~cm}$ and nominal LiDAR point density is $\sim 6$ points $/ \mathrm{m}^{2}$ for each flight line. 12 GCP's were measured by static DGPS.

LiDAR point cloud has been filtered to separate ground, building roofs and vegetation points. Images have been triangulated with SocetSet/ Bingo, including self calibration for camera parameters. Bundle adjustment was run using GCP's and GPS-IMU direct georeferencing. Finally, an orthomosaic was computed with a pixel size of $10 \mathrm{~cm}$.

\subsection{Results and analysis}

\subsubsection{Aerial triangulation}

The Swinglet camera parameters have been scaled to $35 \mathrm{~mm}$ standard. This means that the pixel size has been chosen at 9 $\mu \mathrm{m}$ instead of $1.54 \mu \mathrm{m}$ and image size changed to $36 \mathrm{x} 27 \mathrm{~mm}$. This scaling permit to compare image residuals with the reference camera (also a pixel size of $9 \mu \mathrm{m}$ ).
The aerial triangulation was performed using 2212 tie points and 12 GCP's.

The table one shows the camera calibration parameter for both approaches.

\begin{tabular}{|c|c|c|}
\hline & SocetSet/BINGO & Pix4D \\
\hline Focal c [mm] & 29.073 & 29.725 \\
\hline PPA X/Y [mm] & $-0.022 / 0.020$ & $-0.210 /-0.137$ \\
\hline
\end{tabular}

Table 1: Swinglet camera parameters

The figures 4 show the image residual deformation without and with non radial distortion correction (additional parameters of Bingo). The non radial distortions are very important especially in the centre of the image (about $20 \mu \mathrm{m}$ ). The corrections with additional parameters reduce it to $\sim 4 \mu \mathrm{m}$ in the centre but peripheral residuals remains at $2 / 3^{\text {rd }}$ of the image of $\sim 8 \mu \mathrm{m}$. This leads to stereoscopic imperfection such as residual parallaxes and prevent from doing accurate stereo-plotting.
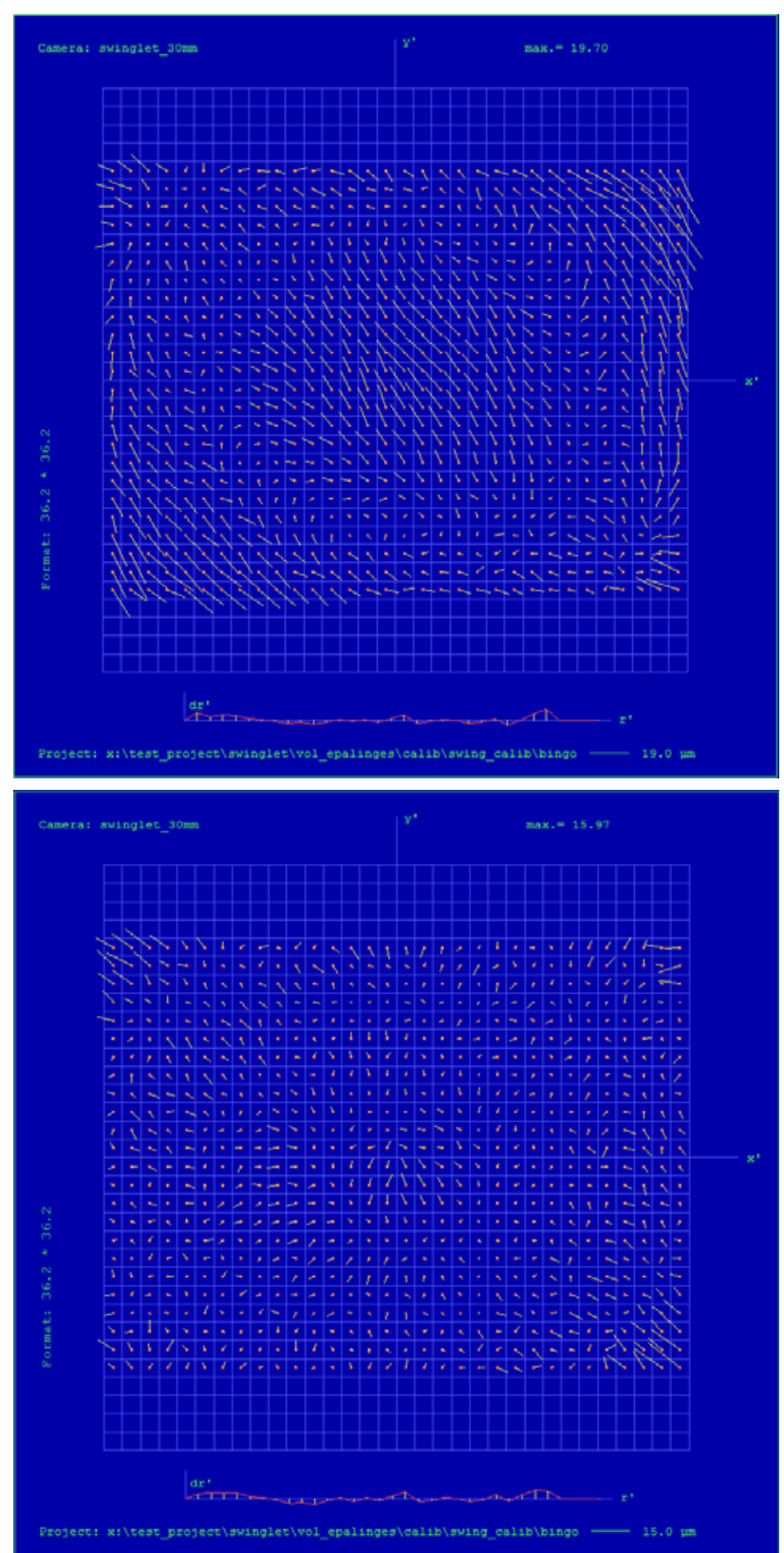


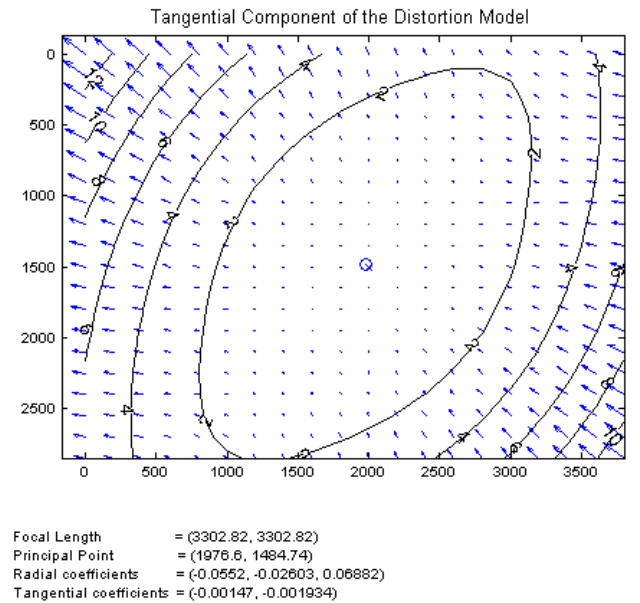

Figure 4: On the top, the residual deformation due to tangential distortions without any correction by additional parameters. In the middle, the same deformation after correction by Bingo parameters. At the bottom, the tangential distortion of PiX4D calibration but the images residuals grid was not available to compare with Bingo grid.

The Table 2 depicts the results of aerial triangulation in terms of object space accuracy with estimated precision of tie point coordinates and GCP residuals. Reference flight (AT-Ref) is also included in the results. The Pix4D report provides only information on GCP's and average image residuals* instead of standard deviation of image residuals $\left(\sigma_{0}\right)$.

\begin{tabular}{|l|c|c|c|c|c|c|c|}
\hline & \multirow{2}{*}{$\begin{array}{c}\sigma_{0} \\
{[\mu \mathrm{m}]}\end{array}$} & \multicolumn{3}{|c|}{ RMS of object points } & \multicolumn{3}{|c|}{ RMS GCP residuals } \\
\cline { 3 - 8 } & & $\mathrm{X}$ & $\mathrm{Y}$ & $\mathrm{Z}$ & $\mathrm{X}$ & $\mathrm{Y}$ & $\mathrm{Z}$ \\
\hline AT-ref & 3.3 & 27 & 26 & 46 & 23 & 24 & 25 \\
\hline AT- Socet & 4.9 & 37 & 38 & 75 & 45 & 30 & 49 \\
\hline AT- Pix4D & $6.5^{*}$ & na & na & na & \multicolumn{3}{|c|}{20 (combined) } \\
\hline
\end{tabular}

Table 2: Accuracy indicator of Bundle adjustment in the image space $\left(\sigma_{0}\right)$ and in the object space.

\subsubsection{DTM extraction}

The comparison has been made between the Pix4D DTM, the SocetSet NGATE DTM and the reference LiDAR DTM/DSM. The figures 5 to 7 show the height difference between the different extracted elevation models and the LIDAR reference.

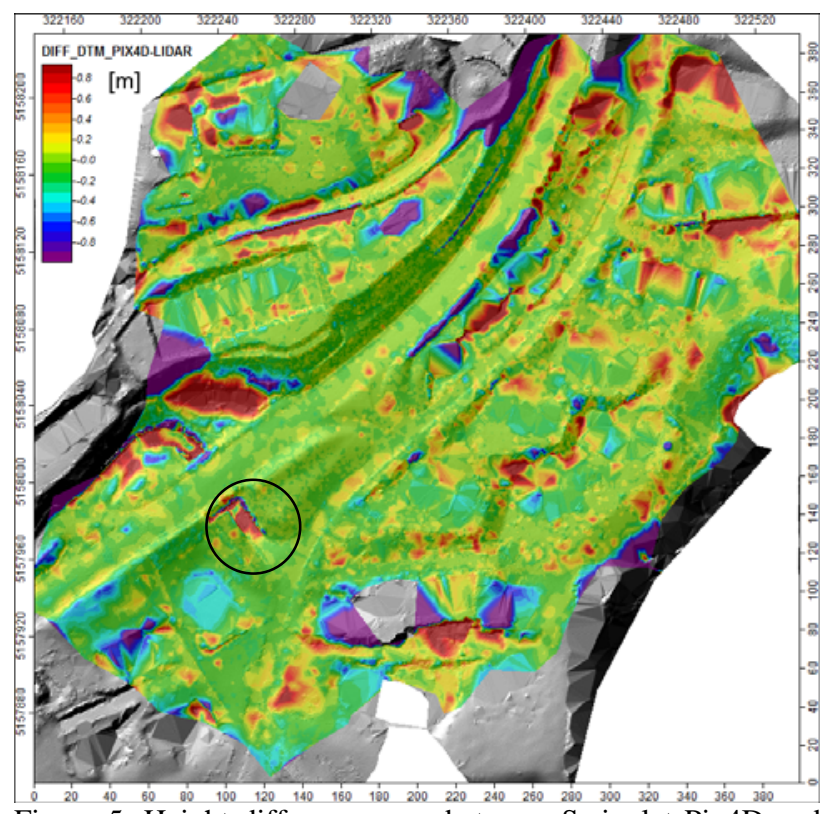

Figure 5: Height difference map between Swinglet Pix4D and LiDAR DTM. On smooth and flat areas, the differences do not exceed $+/-15 \mathrm{~cm}$. As soon as terrain discontinuity appears, the difference increases because the image matching tends to smooth obstacle (buildings, walls, cars). The road below the bridge illustrates this phenomenon (circle). The vegetation areas show also large discrepancies. It is normal since the photogrammetric measurements can not get information below canopy while LiDAR provides it.

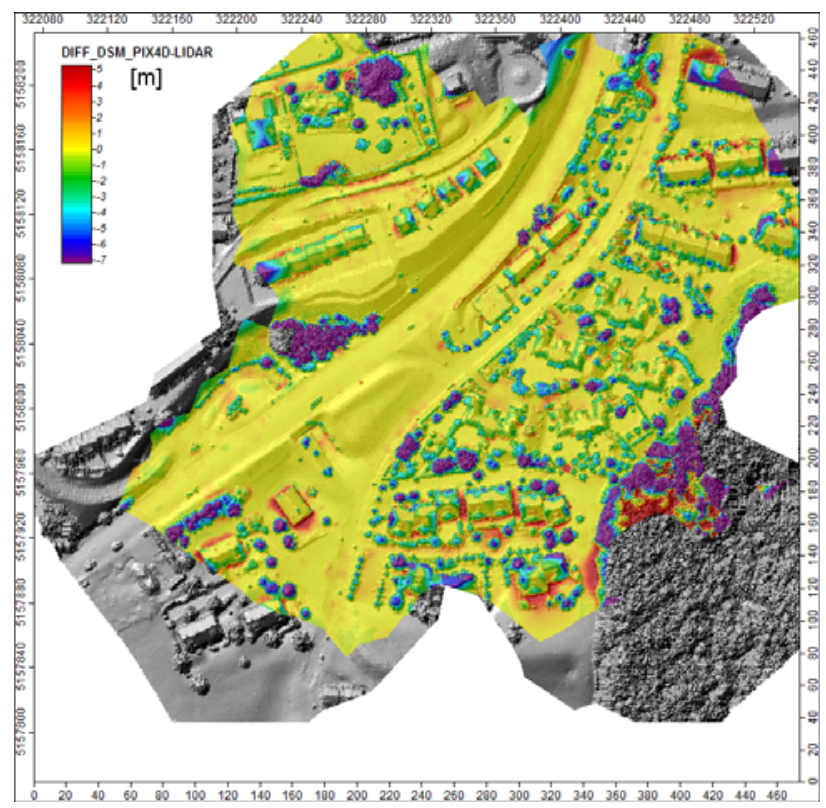

Figure 6: Height difference map between Swinglet Pix4D and LiDAR DSM. This graphic shows also the effect of smoothing near buildings. Nevertheless, the roofs are also within $10 \mathrm{~cm}$ accuracy. The vegetation areas illustrate large differences which can be explained by the period of each flight (March for Swinglet, June for LiDAR). 


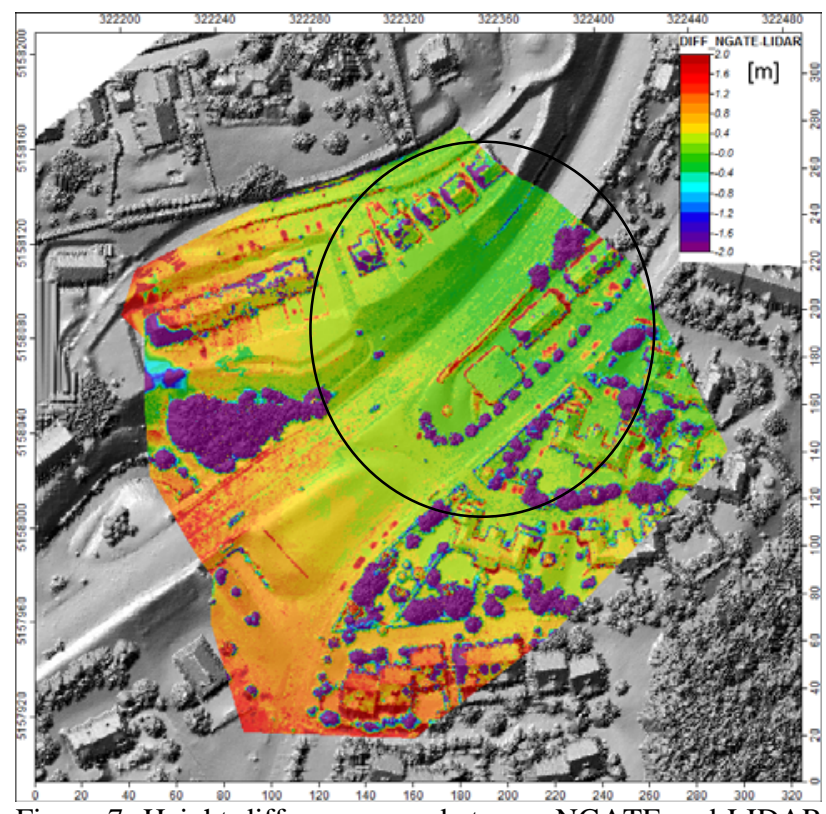

Figure 7: Height difference map between NGATE and LIDAR DSM. The circle fence the area where GCP's are located. In this area, the height difference on smooth terrain ranges of $40 \mathrm{~cm}$. On the left side of the graphics, the difference increases with the distance to the GCP's area to reach $\sim 1.5 \mathrm{~m}$. Moreover, the NAGTE model looks noisy especially in pavement. This can be explained by residual parallaxes observed in the Swinglet models (due to un-modelled deformations) combined to the low contrast of pavement and repeated painted lines. Nevertheless, it is surprising since NGATE generally gives good results even in low contrast surfaces.

Using the same number of GCP's, the Pix4D DTM is more accurate, less noisy than the SocetSet one. The aerial triangulation and the determination of the camera is probably the origin of such difference. The Figure 8 represents the height difference between 1920 ground Tie points (SocetSet) and LiDAR. The magnitude of difference is in the same range than for the DTM (fig. 7).

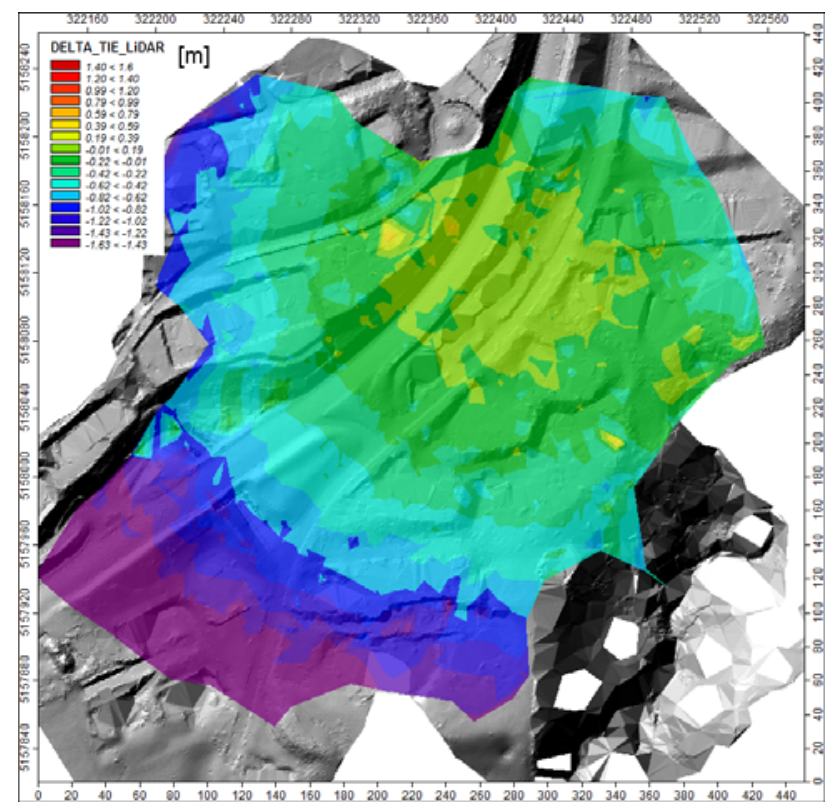

Figure 8: Height differences between SocetSet tie points (on ground) and LiDAR DTM.
The height drift of the Bingo block in the area without GCP's is due to the poor absolute reference while freeing all internal camera parameters. It seems that in this case, depending on the sequence of the parameters are freed (focal length, principal point and radial and tangential distortion), the results in the area without control points can varies significantly on the projection centre coordinates. The Pix4D block keeps consistency with LiDAR in the all area.

Finally, we compared the DTM with GCP's coordinate. The table 3 shows the height difference statistics for each model, including reference data.

\begin{tabular}{|c|c|c|c|c|}
\hline$[\mathrm{m}]$ & Average & $\begin{array}{c}\text { Standard. } \\
\text { deviation }\end{array}$ & Min & Max \\
\hline LiDAR & 0.007 & 0.040 & -0.085 & 0.047 \\
\hline Pix4D & 0.047 & 0.095 & -0.073 & 0.223 \\
\hline NGATE & 0.110 & 0.267 & -0.093 & 0.835 \\
\hline
\end{tabular}

Table 3: Statistics of height difference between DTM's and GCP's. This observation confirm the previous observation with an accuracy (1 sigma) of $\sim 10 \mathrm{~cm}$ for the Swinglet Pix4D DTM and of $\sim 30 \mathrm{~cm}$ for the Swinglet NAGTE DTM

\subsubsection{Orthophoto}

All orthophotos have been computed with the same ground sample dimension (GSD) of $10 \mathrm{~cm}$.

The orthomosaics have been analysed according 3 ways:

- Aspect analysis: checking of geometry of straight line and artefact

- Geometric comparison by digitizing ground elements

- $\quad$ Planimetric accuracy related to GCP’s

The figure 10 shows the aspect difference of the 3 orthophotos. One can notice that either on SocetSet or Pix4D, painted lines are not straight while they are on the reference image. For the SocetSet image, it is mainly due to the high noise of the DTM on the pavement which create deformation in the lines. For Pix4D image, the artefacts are harder to explain since the DTM is consistent with LiDAR. It is probably due to mosaicing technique.

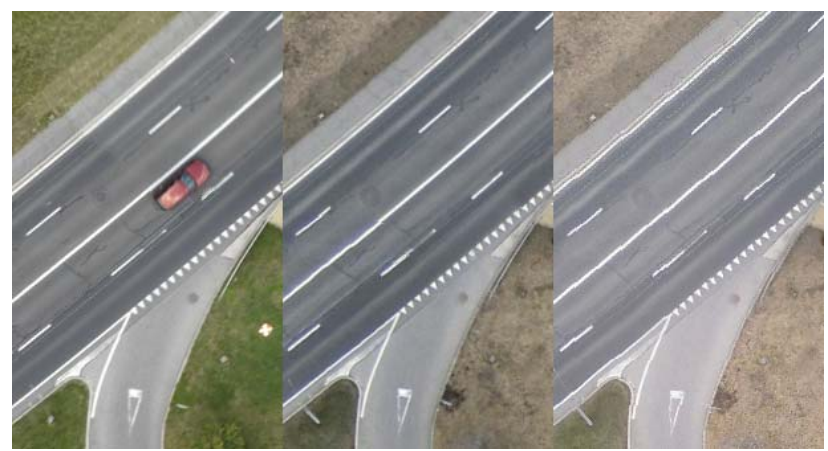

Figure 9: Aspect of orthoimage. Left: reference, Middle: SocetSet, Right: Pix4D

The figure 10 shows the magnitude of planimetric differences between the 3 images. While the SocetSet image is consistent with the reference image, the Pix4D image shows displacement of $\sim 10-15 \mathrm{~cm}$ in several areas. 

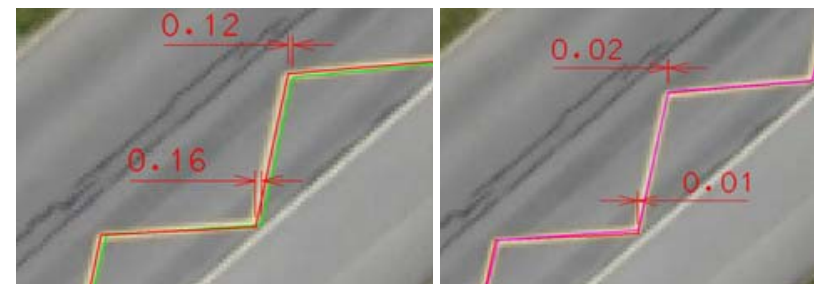

Figure 10: Comparison between feature lines. In Red, reference Orthophoto, in Green, Pix4D Orthophoto, in pink (right) SocetSet orthophoto.

The final comparison is illustrated in the table 4 with GCP's Planimetric residuals. Similarly to features analysis, The Pix4d images shows systematic shift.

\begin{tabular}{|c|c|c|c|c|}
\hline \multirow{2}{*}{} & \multicolumn{2}{|c|}{ Socet set } & \multicolumn{2}{c|}{ Pix4D } \\
\cline { 2 - 5 } & $\mathrm{X}$ & $\mathrm{Y}$ & $\mathrm{X}$ & $\mathrm{Y}$ \\
\hline Average & -0.02 & 0.03 & 0.10 & -0.06 \\
\hline RMS & 0.04 & 0.04 & 0.07 & 0.05 \\
\hline
\end{tabular}

Table 4: Residuals statistics on control points

\section{CONCLUSION AND OUTLOOKS}

The use of Ultra light UAV combine to compact digital camera allow to provide mapping products such as Digital Elevation Model (terrain or surface) and orthoimages. The tested "Swinglet" is an interesting concept with a global weight below $500 \mathrm{~g}$. The lightness and ease of use of the wing offer a good flexibility to map quickly small areas. At the moment, there is apparently no regulation for such "toy" in Switzerland.

The quality of the mapping products issued from this system seems to depend on the processing tools used. The use of standard photogrammetric software provides elevation model with an accuracy of $\sim 30 \mathrm{~cm}$ within GCP's area. The accuracy level seems to be limited by the weakness of modelling correctly the lens distortion with poor quality optics. Standard additional parameters do not handle such big deformation. This results in residual parallaxes of $20-30 \mathrm{~cm}$ in models. This prevent from doing any accurate stereo-plotting. Coventionnal photogrammetric softwares also require more effort for the Tiepoint matching which is poorly automated with such images while computer vision approach is highly automated and successful matching rate is better than $97 \%$.

New approach of multi-image correlation seems to provide better results with such camera. The accuracy of DTM is in the range of $10-15 \mathrm{~cm}$ at a flying height of $150 \mathrm{~m}$.

Finally the orthoimage resulting from the Swinglet images is in both processing ways spotted of artefacts due to DTM artefacts or mosaicing. In both case, the quality of the orthoimage is not reaching the level of the reference flight.

As stereo plotting is not a negligible part of photogrammetry, next investigation will focus on a way to correct distortions directly on the image and on other type of processes such as the open source suite from IGN (Apero-MicMac). It will be also of interest to check the camera stability between 2 successive flights.

\section{REFERENCES}

Bingo 6.0 User manual, 2011.
Eisenbeiss, H., Stempfhuber, W. and Kolb, M., 2009. Genauigkeitsanalyse der 3D-Trajektorie von Mini-UAVs, In: Zukunft mit Tradition "29. Wissenschaftlich-Technische Jahrestagung der DGPF", Ed.: Seyfert, E., Deutschen Gesellschaft für Photogrammetrie, Fernerkundung und Geoinformation (DGPF) e.V., Potsdam, 407-417.

Haala N., 2009. Comback of digital image matching. Photogrammetric Week 2009, Wichmann Verlag, Heidelberg, 289-301.

Next-Generation Automatic Terrain Extraction (NGATE). Innovation in the cost-effective derivation of elevation data from imagery, 2008.

http://www.socetgxp.com/docs/education/white_papers/wp_nga te.pdf

Tola E., Lepetit V., and Fua P. Daisy, 2010. An Efficient Dense Descriptor Applied to Wide Baseline Stereo. IEEE Transactions on Pattern Analysis and Machine Intelligence, 32(5):815- 830.

Vallet J., 2007 GPS-IMU and Lidar integration to aerial photogrammetry: Development and practical experiences with Helimap System ${ }^{\circledR}$, Voträge Dreiländertagung 27. Wissenschaftlich-Technische Jahrestagung der DGPF. 19-21 June 2007, Muttenz.

Wendel, J., Meister, O., Schlaile, C. and Trommer, G. F., 2006. An integrated GPS/MEMS-IMU navigation system for an autonomous helicopter, In: Aerospace Science and Technology, $10,527-533$.

Zufferey, J-C., Beyeler A. and Floreano D., 2010. Autonomous flight at low altitude using light sensors and little computational power. International Journal of Micro Air Vehicles, vol. 2, num. 2, p. 107-117. 